\title{
DCIS of the Breast: A Look towards Discovery and Advancements in the Field
}

\author{
Henry Mark Kuerer, MD, PhD, FACS, ${ }^{1,2,3}$ Lisa S. Wiechmann, MD, ${ }^{1,2}$ and \\ Lajos Pusztai, MD, $\mathrm{PhD}^{1,2}$ \\ ${ }^{1}$ Departments of Surgical Oncology (HMK), Breast Medical Oncology (LP), The University of Texas M. D. Anderson Cancer \\ Center, Houston, TX, USA \\ ${ }^{2}$ Baylor Breast Center (LSW), Baylor College of Medicine, Houston, TX, USA \\ ${ }^{3}$ Department of Surgical Oncology, Unit 444, The University of Texas M. D. Anderson Cancer Center, 1515 Holcombe Blvd., \\ Houston, TX 77030, USA
}

So many assumptions and questions remain regarding ductal carcinoma in situ (DCIS). We overtreat the majority of patients with DCIS as this entity is generally not immediately life threatening. For many patients with an initial diagnosis of DCIS, progression to invasive cancer (if at all) may be over a period of several decades. What are our treatments for DCIS? We perform surgery, utilize radiation in most, use Tamoxifen in many cases, ${ }^{1}$ and we are even beginning trials to test other novel systemic biologic agents for the disease. ${ }^{2,3}$ However, the use of the word "treatment" is probably our first and most obvious mistake when we talk about DCIS. We really need to begin to talk about prevention when we mention DCIS. We treat DCIS to prevent invasive breast cancer; just as we may treat patients with very strong family histories or known genetic predisposition to cancer, or LCIS, or atypical hyperplasia. Our fundamental understanding of the biology and clinical behavior of DCIS is not clear.

Some essential questions regarding DCIS need to be examined systematically:

1. Can we identify patients with DCIS who are the most likely to develop invasive cancer?

2. With no additional therapy, what is the true incidence of development of invasive cancer

Received June 4, 2007; accepted June 5, 2007; published online: August 21, 2007.

Address correspondence and reprint requests to: Henry Mark Kuerer, MD, PhD, FACS; E-mail: hkuerer@mdanderson.org

Published by Springer Science+Business Media, LLC @ 2007 The Society of Surgical Oncology, Inc. following the diagnosis of DCIS based on the age of our patients at diagnosis and other yet to be defined biologic parameters?

3. Can we eliminate all "treatments" for DCIS and follow certain patient subsets with exceptionally good prognosis based on known and yet to be defined biologic parameters?

4. Can we identify a true molecular signature that will accurately differentiate cases of DCIS that can never harm our patients from potentially aggressive DCIS?

5. Can we safely tailor treatment modalities including surgery, radiation therapy, and endocrine treatment for individual patients using clinical and biological information of the disease?

6. What imaging modalities can be developed that will help us best visualize those at risk for concurrent invasive cancer or demonstrate transition from DCIS to invasive disease conclusively on subsequent follow up?

7. What are the immunologic mechanisms present in healthy patients with DCIS that are likely to be at work in the prevention of the development invasive breast cancer?

8. What strategies are needed at a national and global level to facilitate answering these questions and move the field forward?

In "Correlation of Levels and Patterns of Genomic Instability with Histologic Grading of DCIS," Ellsworth et al. ${ }^{4}$ amplified a panel of 52 microsatellite markers, genotyped, and analyzed them to poten- 
tially develop DCIS genomic patterns that correlate with clinical and pathologic classification of DCIS. The investigators found patterns of allelic instability to add further support to previous reported hypothetical models in which DCIS develops along two discreet pathways. Specifically, Ellsworth et al. ${ }^{4}$ have shown that physical loss of chromosome 16q appears essential for the development of well-differentiated DCIS, while allelic instability at 6q, 25q27, 8q24, 9 p21, 13q14, and 17 p13.1 are associated with the development of poorly differentiated lesions.

This study advances our understanding of the genetic changes that are associated with the histological appearance of disease but fails to answer the question of which genetic changes can predict those patients at risk for development of invasive breast cancer. However, the value of these findings should not be underestimated as they are likely a bridge to further advancements in the field. The precise relationship between DCIS and invasive breast cancer remains to be elucidated. The advent and widespread use of screening mammography has led to increasing detection of DCIS in the USA and detection rates of this entity are expected to increase exponentially as the our more healthy global population of women increase their life expectancy and as more developing countries begin screening programs. The hunt for molecular markers or signatures that will accurately predict the nature of the DCIS progression to invasive breast cancer has been extremely challenging and limited primarily by the lack of large repositories of tissue samples and extensive clinical follow-up. A critical need exists for a concerted national program of patients with DCIS, clinicians, and basic scientists to identify molecular parameters of DCIS associated with an actual risk to the life of our patients. The time to advance the field and our understanding of DCIS is now.

\section{REFERENCES}

1. Yen TW, Kuerer HM, Ottesen RA, et al. Impact of randomized clinical trials results on the use of Tamoxifen following breast surgery for ductal carcinoma in situ in the national comprehensive cancer network. J Clin Oncol, 2007; in press.

2. Gonzalez RJ, Buzdar AU, Kuerer HM. Novel clinical trial designs for treatment of ductal carcinoma in situ of the breast with trastuzumab (Herceptin). Breast $J$ 2007; 13 (1):72-75.

3. Czerniecki BJ, Koski GK, Koldovsky U, et al. Targeting HER$2 /$ neu in early breast cancer development using dendritic cells with staged interleukin-12 burst secretion. Cancer Res 2007; 15; 67(4):1842-52.

4. Ellsworth RE, Ellsworth DL, Love B, et al. Correlation of levels and patterns of genomic instability with histologic grading of DCIS. Ann Surg Onc 14; doi:10.1245/s10434-007-9459-8 [Online: June 5, 2007]. 